\title{
ELETRODOS MODIFICADOS COM POLÍMEROS PERFLUORADOS E SULFONADOS: APLICAÇÕES EM ANÁLISES AMBIENTAIS
}

\author{
Denise Alves Fungaro \\ Divisão de Diagnóstico Ambiental do Departamento de Engenharia Química e Ambiental - Instituto de Pesquisas Energéticas e \\ Nucleares - Travessa R, 400 - Cidade Universitária - 05508-900 - São Paulo - SP \\ Christopher M. A. Brett \\ Universidade de Coimbra - Departamento de Química, 3049 - Coimbra - Portugal \\ Recebido em 13/10/99; aceito em 4/2/00

\begin{abstract}
PERFLUORINATED AND SULFONATED POLYMERS MODIFIED ELECTRODES: APPLICATIONS TO ENVIRONMENTAL ANALYSIS. Perfluoro and sulfonated ion-exchange polymers are recognized as a very useful material for various mechanistic studies and applications in electrochemistry. These polymers are characterized by high equivalent weights and by a low number of ion-exchange sites interposed between long organic chains. The solubility enables a preparation of stable polyelectrolyte films on the electrode surface. Examples of the determination of trace metals and organic componds in real environmental samples are presented.
\end{abstract}

Keywords: ion-exchange voltammetry; polymeric coating; electroanalysis.

\section{INTRODUÇÃO}

A capacidade de pré-concentração dos eletrodos modificados com filmes finos de polímeros de troca iônica foi descrita em estudos do começo da década de $80^{1-3}$. A técnica que utiliza estes sensores recebeu a denominação de voltametria de troca iônica $(\text { IEV })^{4}$ apresentando inúmeras potencialidades e aplicações analíticas.

Nesta técnica usam-se eletrodos sólidos, geralmente, carbono vítreo, grafite pirolítico, platina ou ouro, coberto com um filme fino do polímero trocador de íon, o qual permite uma pré-concentração, detecção voltamétrica simultânea e rápida do íon analito eletroativo. O sinal voltamétrico registrado nestas condições depende da concentração das espécies eletroativas incorporadas pelo trocador iônico dentro da camada poliméri$\mathrm{ca}^{5}$. Se um trocador iônico caracterizado pela seletividade própria for usado, é possível determinar analitos eletroativos iônicos em níveis de concentração de submicromolar. Além do mais, a presença da cobertura poliônica na superfície do eletrodo evita fenômeno de adsorção ou precipitação. Estes fenômenos observados frequentemente na análise de matrizes complexas, como fluidos biológicos ou amostras ambientais ${ }^{6-7}$, podem desativar a superfície do eletrodo e impedir a aplicação direta dos métodos voltamétricos em amostras reais.

A utilização da pré-concentração de troca iônica em conjunto com as técnicas eletroquímicas foi estudada também para o desenvolvimento de eletrodos de pasta de carbono modificados pela mistura do aglutinante usado na preparação da pasta com um trocador iônico adequado ${ }^{8-9}$.

Os avanços na avaliação quantitativa e físico-química do comportamento eletroquímico dos eletrodos modificados com polímeros encontram-se descritos em artigos de revisão na literatura $^{10-13}$. Alguns aspectos das aplicações analíticas da voltametria de troca iônica em eletroanálise foram discutidos enfocando o contexto como método de pré-concentração nãofaradaico $^{5}$ ou como determinação eletroquímica de metais traço e substâncias orgânicas com eletrodos modificados ${ }^{14-15}$.

Neste artigo são descritas as utilizações mais comuns dos eletrodos modificados com polímeros de troca iônica perfluorados e

e-mail : dfungaro@ net.ipen.br sulfonados aplicados na determinação das características eletroquímicas de espécies em amostras ambientais.

\section{PRINCÍPIOS}

A vantagem de cobrir a superfície de eletrodo com um filme fino de um polímero de troca iônica vem da possibilidade de explorar o equilíbrio de troca iônica que se estabelece na interface polímero-solução como uma pré-concentração nãofaradaíca dos contra-íons eletroativos. Obtém-se sensibilidades mais altas acompanhadas de inerente aumento da seletividade. A detecção pode ser realizada diretamente ou na interface polímeroeletrodo via uma medição voltamétrica ou amperométrica.

Os princípios serão discutidos considerando-se a pré-concentração e a detecção eletroquímica redutiva de um analito catiônico oxidado num eletrodo coberto com um filme polianiônico. Entretanto, os mesmos princípios podem ser considerados, com as devidas correções, para a voltametria de troca iônica de espécies aniônicas oxidadas em um eletrodo coberto com filme policatiônico e para analitos catiônicos ou aniônicos reduzidos.

A capacidade de pré-concentração de uma cobertura polimérica é regida pelo equilíbrio de troca iônica entre o contra-íon eletroativo (analito) e os contra-íons não eletroativos presentes em solução ou já incorporados na cobertura. A reação de troca iônica de um polímero de troca catiônica é a seguinte ${ }^{16}$.

$\mathrm{O}^{\mathrm{m}+}+\mathrm{m}\left(\mathrm{P}^{-} \mathrm{X}^{+}\right) \times \mathrm{P}_{\mathrm{m}}^{-} \mathrm{O}^{\mathrm{m+}}+\mathrm{mX}^{+}$

onde $\mathrm{P}^{-}=$sítios de troca iônica no polímero; $\mathrm{X}^{+}=$contra-íon inerte eletroquimicamente (considerando um cátion monocarregado, o qual geralmente é do eletrólito suporte); $\mathrm{O}^{\mathrm{m+}}=$ contra-íon multicarregado eletroativo (analito).

A espécie $\mathrm{O}^{\mathrm{m}+}$ pode ser reduzida na interface polímero-eletrodo gerando um sinal voltamétrico, de acordo com a reação:

$\mathrm{P}^{-} \mathrm{m}^{\mathrm{m}+}+\mathrm{ne}^{-}+\mathrm{n} \mathrm{X}^{+} \times\left(\mathrm{P}^{-}\right)_{(\mathrm{m}-\mathrm{n})} \mathrm{O}^{(\mathrm{m}-\mathrm{n})+}+\mathrm{n}\left(\mathrm{P}^{-} \mathrm{X}^{+}\right)$

Como o processo ocorre na interface polímero-eletrodo, a corrente de redução é uma função da concentração das espécies eletroativas incorporadas na cobertura polimérica e da velocidade de transporte de eléctron dentro da cobertura ${ }^{17}$. 
Ugo et al. ${ }^{18}$ discutiram os resultados de estudos mecanísticos relevantes para o desenvolvimento e otimização dos métodos IEV.

\section{PARÂMETROS PARA UTILIZAÇÃo DOS POLÍMEROS}

\section{Escolha do polímero e reutilização das coberturas}

A escolha de um trocador iônico para ser usado como modificador de um eletrodo tem que levar em consideração as propriedades de troca iônica da cobertura polimérica e a facilidade de obtenção de um filme polimérico estável e reprodutível na superfície eletródica.

A influência de parâmetros na estrutura, estabilidade e comportamento de troca iônica das coberturas poliméricas foram estudas em relação à composição da solução polimérica e temperatura de secagem ${ }^{19-22}$, umidade relativa ${ }^{23}$ ou tratamento do polímero com plasma ${ }^{24}$.

A reutilização (regenerabilidade) da superfície do eletrodo modificado é um ponto importante nas aplicações analíticas da voltametria de troca iônica. Se o coeficiente de seletividade para o analito não é muito grande, a regeneração pode ser alcançada pela simples exposição do filme à uma solução salina não eletroativa de alta concentração para obter a expulsão dos analitos do filme trocador iônico. Entretanto, este procedimento simples não pode ser usado com analitos caracterizados por coeficientes de seletividade altos.

\section{Métodos de preparação do eletrodo}

A modificação da superfície do eletrodo com polímero ocorre com muitas monocamadas de espécies ativas. Tipicamente, os polímeros empregados tem sítios quimicamente ou eletroquimicamente ativos e respostas eletroquímicas maiores e mais fáceis são obtidas quando grandes quantidades de camadas são colocadas na superfície do eletrodo. Vários métodos são usados para preparar eletrodos cobertos com filmes poliméricos:

1. cobertura por banho: um eletrodo é mantido em uma solução do polímero, a qual é depositada via um processo de adsorção.

2. evaporação: um pequeno volume da solução polimérica é colocada sobre a superfície do eletrodo e evapora-se o solvente. O eletrodo é usado em um eletrólito ou solvente onde o polímero é insolúvel.

3. deposição oxidativa ou redutiva: depende da solubilidade do polímero no estado iônico. O polímero oxidado (ou reduzido) em um solvente resulta em uma forma insolúvel que precipita na superfície do eletrodo.

4. cobertura e rotação: o eletrodo é mantido girando após uma gota da solução polimérica ter sido colocada na sua superfície.

5. eletropolimerização: os produtos da reação no eletrodo são poliméricos e insolúveis no solvente usado. A eletropolimerização de vários compostos monoméricos substituídos pode proporcionar ao eletrodo propriedades elétricas e analíticas interessantes.

6. polimerização por métodos não eletroquímicos: polimerização ativada por plasma, foto-induzida por UV ou polimerização de organosilanos.

Embora as respostas eletroquímicas dos eletrodos modificados por polímeros sejam mais fáceis de detectar do que aquelas dos eletrodos modificados por monocamadas, elas também serão complicadas pela sobreposição de processos como transporte e transferência de carga, movimento de contra-íons para dentro e fora do polímero, movimento do filme polimérico e inchaço com o solvente e difusão do analito no filme. Estes processos foram estudados para melhorar a interpretação da resposta voltamétrica ${ }^{25}$. A despeito destes fatos, a modificação polimérica do eletrodo permanece um procedimento conveniente e simples para melhorar o desempenho de eletrodos apresentando uma infinidade de aplicações.

\section{IONÔMEROS PERFLUORADOS E SULFONADOS}

Um ionômero é um polímero linear ou ramificado, o qual contém grupos ionizáveis covalentemente ligados. Eles geralmente contém pequenas quantidades de grupos ionizáveis (menos que $10 \%$ por mol do monômero ionizável) e não estão covalentemente ligados por ligação cruzada. Assim, os ionômeros são quimicamente e morfologicamente distintos dos demais materiais orgânicos trocadores de íon.

Os ionômeros perfluorados e sulfonados mais comuns são Nafion, Tosflex e Eastman AQ que são caracterizados por massas equivalentes elevadas e um número baixo de sítios trocadores de íons interpostos entre longas cadeias orgânicas. A estrutura da cobertura solvatada apresenta uma parte hidrofóbica e uma parte hidrofílica ${ }^{26}$, onde a zona hidrofóbica é responsável pela adsorção e estabilização das coberturas nos materiais hidrofóbicos dos eletrodos como carbono vítreo e grafite pirolítico.

A membrana Nafion foi desenvolvida para a produção eletrolítica de cloro pela empresa norte americana DuPont (Figura 1). $\mathrm{O}$ material da membrana consiste de um polímero perfluorado com unidades de tetrafluorpolietileno $(n=5$ a 13), ligado a um éter, que por sua vez está ligado a um ácido sulfônico.

$$
\begin{gathered}
{\left[-\left(-\mathrm{CF}_{2}-\mathrm{CF}_{2}-\right)_{\mathrm{n}}-\mathrm{CF}_{2}-\mathrm{CF}-\right]_{\mathrm{x}}} \\
\mid \\
\left(\mathrm{O}-\mathrm{CF}_{2}-\mathrm{CF}-\right)_{\mathrm{m}}-\mathrm{O}-\mathrm{CF}_{2}-\mathrm{CF}_{2}-\mathrm{SO}_{3}^{-}\left(\mathrm{Na}^{+}, \mathrm{H}^{+}\right) \\
\mid \\
\mathrm{CF}_{3}
\end{gathered}
$$

Figura 1. Estrutura do ionômero perfluorado Nafion.

A condutividade da membrana em parte é devida à presença do grupo sulfônico. Por outro lado, a membrana úmida inchase e ocorre um tipo de separação de fases, onde o grupo perfluorado forma uma matriz hidrófoba, os éteres formam uma fase intermediária até o grupo sulfônico, que por sua vez forma uma terceira fase hidrofílica, na qual a água está absorvida. Nesta terceira fase, a água apresenta fortes pontes de hidrogênio e também encontram-se os cátions, como $\mathrm{Na}^{+} \mathrm{e} \mathrm{H}^{+}$. A condutividade iônica da membrana baseia-se principalmente na solvatação dos prótons e na existência da fase hidrofílica.

A dissolução do filme polimérico de Nafion e as características eletroquímicas do eletrodo coberto com o filme foram primeiramente estudados por Martin et al. ${ }^{27}$ e posteriormente surgiu um grande número de estudos.

O Nafion apresenta uma enorme afinidade por cátions orgânicos hidrofóbicos conforme atestam os coeficientes de seletividade determinados por Espenscheid et al. ${ }^{4}$. A pré-concentração máxima irá ser obtida quando o equilíbrio filme-solução do analito for alcançado. $\mathrm{O}$ tempo requerido para o estabelecimento do equilíbrio depende do tamanho, carga, estrutura e concentração do contra-íon do analito e da força iônica da solução do analito. Em geral, a velocidade do equilíbrio filme-solução é limitada pela velocidade da difusão iônica no filme. As membranas com maior velocidade de transporte de íon irão produzir sensores modificados com tempo de resposta mais rápido.

O polímero poli(éster ácido sulfônico), também conhecido como Eastman AQ, é um trocador catiônico disponível comercialmente (Eastman KodaK Co.) com propriedades semelhantes àquelas do Nafion (Figura 2). A diferença com o Nafion reside na solubilidade. O Eastman $\mathrm{AQ}$ é bem mais insolúvel e utilizam-se dispersões do polímero, geralmente em água ou em água:acetona, para preparação do eletrodo. 


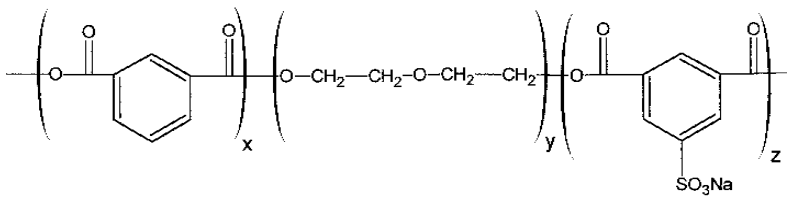

AQ 55

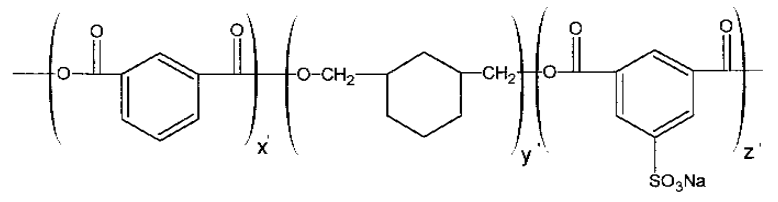

$A Q 29$

Figura 2. Estrutura dos polímeros Eastman AQ 55, AQ 29 e AQ 38.

As massas equivalentes dos três polímeros foram determinados pela porcentagem de sulfonação como 1500 (AQ 55) e 2500 (AQ 38 e AQ 29).

O ionômero Tosflex (TOSOH Co., Ltda., Tokyo, Japão) foi a primeira membrana perfluorada trocadora de ânions disponível comercialmente no mundo. $\mathrm{Na}$ estrutura (Figura 3), m é igual a 0 ou 1 , n é igual a 1 ou 5 e $\mathrm{X}$ denota a unidade trocadora de ânions, incluindo o contra-ânion.

$$
\begin{aligned}
& -\left(\mathrm{CF}_{2}-\mathrm{CF}_{2}-\right)_{\mathrm{x}}-\left(-\mathrm{CF}_{2}-\mathrm{CF}-\right)_{\mathrm{y}}- \\
& \left(\mathrm{OCF}_{2}-\underset{\mathrm{CF}_{3}}{\mathrm{CF}}-\right)_{\mathrm{m}}-\mathrm{O}-\left(-\mathrm{CF}_{2}-\right)_{\mathrm{n}}-\mathrm{X}
\end{aligned}
$$

Figura 3. Estrutura da membrana Tosflex.

Um procedimento para a dissolução não-destrutiva da membrana de Tosflex, formação do filme no eletrodo de carbono vítreo e estudos eletroquímicos básicos foram realizados ${ }^{28}$. Uma solução do polímero em metanol:isopropanol:água (1:1:1) formou um bom filme sobre a superfície do eletrodo.

A possibilidade de utilização de um eletrodo modificado por ionômero irá depender da seletividade em relação ao analito, velocidade de transporte de massa e a regeneração rápida do filme polimérico após término da análise.

\section{APLICAÇÕES EM AMOSTRAS AMBIENTAIS}

Nos parágrafos seguintes e na Tabela 1 são apresentadas algumas das aplicações eletroanalíticas mais comuns dos sensores cobertos com polímeros perfluorados e sulfonados para a determinação de espécies em amostras ambientais.

O primeiro estudo usando eletrodo de carbono vítreo coberto com filme fino de mercúrio e modificado com Nafion (NCTMFE) para determinação de $\mathrm{Cd}, \mathrm{Pb}$ e $\mathrm{Cu}$ por voltametria de redissolução anódica (ASV) foi realizado por Hoyer et al. ${ }^{6}$. A preparação do filme fino de mercúrio na interface de um eletrodo de carbono vítreo envolveu a redução eletroquímica de cátions $\mathrm{Hg}^{2+}$ incorporados pela troca iônica no Nafion. A cobertura de Nafion estabilizou mecanicamente as gotículas de mercúrio formadas por prevenir o deslocamento que pode ocorrer no eletrodo de filme de $\mathrm{Hg}$ não modificado. A sensibilidade do NCTMFE dependeu da espessura do filme. A depressão dos picos na presença de substâncias orgânicas foi testada. A cobertura foi excelente contra proteínas e álcoois. A proteção contra detergentes foi menos eficiente, principalmente para os catiônicos e não iônicos.

Estudos compararam diversos procedimentos de especiação físico-química e a toxicidade de metais para micoorganismos aquáticos ${ }^{29}$. Um dos procedimentos analisados foi a
ASV para a determinação de $\mathrm{Cd}, \mathrm{Pb}, \mathrm{Cu}$ e $\mathrm{Zn}$ em águas ambientais. Usou-se NCTMFE, eletrodo de mercúrio de gota pendente e eletrodo de carbono vítreo coberto com filme fino de mercúrio. Os resultados obtidos com o NCTMFE foram os que mais se aproximaram dos bioensaios. Isto ocorreu porque a difusão dos metais através da cobertura de Nafion é similar à difusão que ocorre através da biomembrana dos microorganismos aquáticos.

A especiação eletroquímica do complexo de $\mathrm{Cu}$ com substâncias orgânicas foi analisada com ASV usando NCTMFE e eletrodo de carbono vítreo coberto com filme fino de mercúrio e modificado com a mistura de Nafion e acetato de celulose $(\mathrm{CANMFE})^{30}$. O CANMFE funcionou como o NCTMFE acrescentando-se a capacidade do acetato de celulose para exclusão de espécies da superfície do eletrodo com base no tamanho. Os complexos negativos eram inertes nos eletrodos cobertos com polímeros e o complexo $\mathrm{Cu}$-ácido fúlvico apresentou a eletroatividade mais baixa no CANMFE. O método foi aplicado na análise de águas poluídas.

O NCTMFE foi preparado pela pré-mistura de uma solução de acetato de mercúrio com uma solução etanólica de Nafion para a determinação de metais traço usando $\mathrm{ASV}^{31}$. O procedimento evitou o uso da técnica em banho tornando a preparação mais fácil e rápida. Determinou-se $\mathrm{Pb}$ e $\mathrm{Cu}$ em efluentes de galvanoplastia usando-se FIA. O efeito de surfactantes na faixa de 1 a $100 \mathrm{mg} \mathrm{L}^{-1}$ foi observado e obteve-se os mesmos resultados dos estudos anteriores ${ }^{6}$.

Um estudo comparativo entre eletrodos de filme fino de mercúrio cobertos com diversos polímeros usados para análise voltamétrica de $\mathrm{Pb}$ e $\mathrm{Cd}$ na presença de surfactantes foi realizado $^{32}$. Entre os eletrodos, o NCTMFE mostrou bom desempenho frente ao dodecil sulfato de sódio e proteína. A proteção mostrou-se inadequada para Triton X-100 na determinação de $\mathrm{Pb}$. $\mathrm{O}$ pico de $\mathrm{Pb}$ aumentava com a adição do detergente porque ocorria uma complexação entre os íons e o surfactante.

A complexação da matéria húmica com o chumbo em água natural foi estudada por ASV usando eletrodo de carbono vítreo coberto com filme fino de mercúrio não modificado e modificado com Nafion ${ }^{33}$. Os resultados mostraram que o Nafion minimizou bastante a adsorção da matéria húmica no eletrodo. O complexo é lábil com o eletrodo de carbono vítreo coberto com filme fino de mercúrio, porém os resultados quanto à labilidade não foram claros com o NCTMFE.

Estudos da especiação do chumbo com o eletrodo de filme fino de mercúrio coberto com Nafion foram realizados ${ }^{34}$. Os agentes complexantes investigados foram: ácido nitriloacético, etilenodiamina, ácido oxálico e ácido húmico. As constantes de estabilidade dos complexos foram determinadas usando-se o eletrodo de filme fino de mercúrio não modificado e modificado com o polímero.

As características da ASV com NCTMFE usando irradiação ultra-sônica foi estudada em detalhes ${ }^{35}$. O principal efeito do ultra-som é aumentar bastante o transporte de massa para a pré-concentração dos analitos na superfície do eletrodo e, consequentemente, há um aumento de uma ordem de magnitude na sensibilidade do método. O procedimento de preparação do NCTMFE foi otimizado. O limite de detecção para ambas as espécies $\mathrm{Pb}$ e $\mathrm{Cd}$ em água foi $3 \times 10^{-11} \mathrm{~mol} \mathrm{~L}^{-1}$ com préconcentração de 30 segundos.

Wang et al. ${ }^{36}$ determinaram $\mathrm{Pb}, \mathrm{Cd}$ e $\mathrm{Cu}$, na presença de surfactantes, utilizando análise voltamétrica e eletrodo de filme fino de mercúrio coberto com Eastman AQ. A corrente dos metais não sofreu alteração na presença de gelatina, Triton $\mathrm{X}$ 100, albumina e ácido húmico até $100 \mathrm{mg} \mathrm{L}^{-1}$.

O estudo e a caracterização de eletrodos cobertos com trocadores aniônicos tem sido realizados com compostos modelos redox, porém há poucos estudos eletroanalíticos. A maioria das aplicações envolvem a determinação de metais em águas na forma de seus complexos aniônicos. 
Tabela 1. Exemplos de IEV com eletrodos cobertos com polímeros perfluorados e sulfonados.

\begin{tabular}{|c|c|c|c|c|c|}
\hline Analito & Eletrodo & Polímero & Características & Técnica & Ref. \\
\hline $\mathrm{Pb}, \mathrm{Cd}$ & $\mathrm{CV}$ & Nafion & $\begin{array}{l}\text { Análise de água } \\
\text { f.t.: } 1 \mathrm{nmol} \mathrm{L} \mathrm{L}^{-1}-2 \mu \mathrm{mol} \mathrm{L} \mathrm{L}^{-1}\end{array}$ & $\begin{array}{l}\text { SWASV } \\
\text { DPASV }\end{array}$ & 6 \\
\hline $\mathrm{Zn}, \mathrm{Cd}, \mathrm{Pb}, \mathrm{Cu}$ & $\mathrm{CV}$ & Nafion & $\begin{array}{l}\text { Análise de águas de rio e } \\
\text { de escoamento } \\
\text { f.t.: } 10^{-7}-0,05 \mathrm{~mol} \mathrm{~L}^{-1}\end{array}$ & DPASV & 29 \\
\hline $\mathrm{Cd}, \mathrm{Pb}, \mathrm{Cu}$ & $\mathrm{CV}$ & Nafion & $\begin{array}{l}\text { Águas naturais. Estudos } \\
\text { de especiação }\end{array}$ & DPASV & 30 \\
\hline $\mathrm{Pb}, \mathrm{Cu}$ & $\mathrm{CV}$ & Nafion & $\begin{array}{l}\text { Efluentes industriais } \\
\text { 1.d.: } \mathrm{Pb} 5 \mathrm{e} \mathrm{Cu} 4 \mu \mathrm{g} \mathrm{L}^{-1} \\
\text { f.t.: } 0,1-1,0 \mathrm{mg} \mathrm{L}^{-1}\end{array}$ & ASV & 31 \\
\hline $\mathrm{Pb}, \mathrm{Cd}$ & $\mathrm{CV}$ & Nafion & $\begin{array}{l}\text { Análise de água } \\
\text { f.t.: } 0,05-0,2 \mu \mathrm{mol} \mathrm{L}{ }^{-1}\end{array}$ & ASV & 32 \\
\hline $\mathrm{Pb}$ & $\mathrm{CV}$ & Nafion & $\begin{array}{l}\text { Águas naturais. Estudo } \\
\text { da complexação com M.H. } \\
\text { f.t.: } 3 \mathrm{nmol} \mathrm{L}^{-1}-1 \mu \mathrm{mol} \mathrm{L}\end{array}$ & DPASV & 33 \\
\hline $\mathrm{Pb}$ & $\mathrm{CV}$ & Nafion & $\begin{array}{l}\text { Análise de água. } \\
\text { Determinação de constantes } \\
\text { de estabilidade. }\end{array}$ & DPASV & 34 \\
\hline $\mathrm{Pb}, \mathrm{Cd}$ & $\mathrm{CV}$ & Nafion & $\begin{array}{l}\text { Águas naturais e efluentes } \\
\text { 1.d.: } 0,03 \mathrm{nmol} \mathrm{L}^{-1} . \\
\text { f.t.: } 5-50 \mathrm{nmol} \mathrm{L}^{-1}\end{array}$ & SWASV & 35 \\
\hline $\mathrm{Cd}, \mathrm{Pb}, \mathrm{Cu}$ & $\mathrm{CV}$ & AQ & $\begin{array}{l}\text { Análise de água } \\
\text { f.t.: } 10-100 \mathrm{nmol} \mathrm{L}-1\end{array}$ & DPASV & 36 \\
\hline $\mathrm{Hg}$ & $\mathrm{CV}$ & Tosflex & $\begin{array}{l}\text { Água do mar } \\
\text { 1.d.: } 0,04 \mathrm{nmol} \mathrm{L}-1 \\
\text { f.t.: } 10^{-7}-10^{-5} \mathrm{~mol} \mathrm{~L}^{-1}\end{array}$ & ASV & 37 \\
\hline $\mathrm{Cu}$ & $\mathrm{CV}$ & Tosflex & Água do mar. 1.d.: $3 \mu \mathrm{mol} \mathrm{L}{ }^{-1}$ & LSASV & 38 \\
\hline $\mathrm{Pb}, \mathrm{Cd}$ & $\mathrm{CV}$ & Nafion & $\begin{array}{l}\text { Águas residuárias } \\
\text { 1.d.: } \mathrm{Cd} 2 \mathrm{e} \mathrm{Pb} 4 \mathrm{nmol} \mathrm{L}^{-1} \\
\text { f.t.: } 5 \times 10^{-9}-10^{-7} \mathrm{~mol} \mathrm{~L}^{-1}\end{array}$ & BIA-ASV & 43 \\
\hline $\mathrm{Zn}, \mathrm{Cd}, \mathrm{Pb}, \mathrm{Cu}$ & RAM & Nafion & $\begin{array}{l}\text { Análise de água } \\
\text { 1.d.: } \mathrm{Cd} 1,3 \text { e } \mathrm{Pb} 1,0 \mathrm{nmol} \mathrm{L}-1 \\
\text { f.t.: } 10^{-9}-10^{-7} \mathrm{~mol} \mathrm{~L}^{-1}\end{array}$ & BIA-ASV & 44 \\
\hline $\mathrm{Zn}, \mathrm{Cd}, \mathrm{Pb}, \mathrm{Cu}$ & $\mathrm{CV}$ & Nafion/pves & $\begin{array}{l}\text { Águas naturais e residuárias } \\
\text { l.d.: } 2-4 \mathrm{nmol} \mathrm{L} \\
\text { f.t.: } 10-60 \mathrm{nmol} \mathrm{L}^{-1}\end{array}$ & BIA-ASV & 45 \\
\hline $\mathrm{Pb}, \mathrm{Cd}$ & $\mathrm{CV}$ & AQ & $\begin{array}{l}\text { Análise de água } \\
\text { l.d.: } 3,4-5,9 \mathrm{nmol} \mathrm{L}^{-1} \\
\text { f.t.: } 10-60 \mathrm{nmol} \mathrm{L}^{-1}\end{array}$ & BIA-ASV & 46 \\
\hline Nitrosaminas & $\mathrm{CV}$ & Nafion & $\begin{array}{l}\text { Análise de água } \\
\text { 1.d.: } 3 \mathrm{nmol} \mathrm{L}-1 \\
\text { f.t.: } 10-60 \mathrm{nmol} \mathrm{L}^{-1}\end{array}$ & CSV & 47 \\
\hline $\mathrm{Pb}$ & Grafite & Nafion/PAN & $\begin{array}{l}\text { Análise de água. } \\
\text { l.d.: } 10 \mathrm{nmol} \mathrm{L}{ }^{-1} \\
\text { f.t.: } 0,05-10 \mu \mathrm{mol} \mathrm{L}^{-1}\end{array}$ & ASV & 48 \\
\hline $\mathrm{Cu}$ & $\mathrm{CV}$ & Nafion/ imidazola & $\begin{array}{l}\text { Águas naturais e residuárias } \\
\text { f.t.: } 10^{-8}-10^{-6} \mathrm{~mol} \mathrm{~L}^{-1}\end{array}$ & DPV & 49 \\
\hline $\mathrm{Cu}$ & Grafite & $\begin{array}{l}\text { Nafion/4- } \\
\text { acilpirazolina }\end{array}$ & $\begin{array}{l}\text { Águas naturais } \\
\text { l.d.: } 1 \mathrm{nmol} \mathrm{L}^{-1} \\
\text { f.t.: } 10^{-9}-10^{-6} \mathrm{~mol} \mathrm{~L}^{-1}\end{array}$ & ASV & 50 \\
\hline
\end{tabular}

$\mathrm{CV}$ = carbono vítreo; RAM = Random Assemblies of Microdisks; 1.d. = limite de detecção; f.t. = faixa de trabalho; M. H. = matéria húmica; pves = polímeros vinílicos e estireno sulfonados; PAN = 1-(2-piridilazo)-2-naftol; SWASV = voltametria de redissolução anódica de onda quadrada; DPASV = voltametria de redissolução anódica de pulso diferencial; ASV = voltametria de redissolução anódica; LSASV = voltametria de redissolução anódica de varredura linear; BIA = análise em fluxo descontínuo; $\mathrm{CSV}=$ voltametria de redissolução catódica; DPV = voltametria de pulso diferencial. 
A determinação de traços de $\mathrm{Hg}^{2+}$ como complexo aniônico tetracloro mercurato com IEV e eletrodo de carbono vítreo coberto com Tosflex foi estudada ${ }^{37}$. A aplicação na análise de água do mar foi descrita. O limite de detecção foi de $4 \times 10^{-11} \mathrm{~mol} \mathrm{~L}^{-1}$.

A determinação de traços de cobre foi realizada via préconcentração de complexos aniônicos de $\mathrm{Cu}^{+}$, eletrogerados in situ na interface eletrodo/polímero Tosflex, pela redução de $\mathrm{Cu}^{2+}$ em meio contendo cloreto ${ }^{38}$. O método pode ser aplicado na análise de água do mar e estudos de especiação e mostrou um desempenho melhor do que a determinação direta de $\mathrm{Cu}^{2+}$ usando eletrodo coberto com Nafion.

A técnica de análise em fluxo descontínuo (BIA) pode ser facilmente combinada com os métodos eletroanalíticos ${ }^{39-42}$. Esta técnica envolve a injeção de uma alíquota de amostra com uma micropipeta em direção ao centro de um eletrodo de disco imerso em um grande volume de solução de eletrólito suporte. Utilizando a voltametria de redissolução anódica em conjunto com a BIA (BIA-ASV) obtém-se quantificação em níveis de $\mu \mathrm{g} \mathrm{L}^{-1} \mathrm{e}$ uma velocidade de injeção de até 60 amostras por hora.

Um benefício particular dos pequenos volumes injetados (< $100 \mu \mathrm{L}$ ) em um grande volume de eletrólito na célula é que o tempo de contato entre amostra e o eletrodo é bastante pequeno. Os problemas de envenenamento do eletrodo são reduzidos quando comparados com outros sistemas. No entanto, para matrizes muito complexas como águas residuárias com altos níveis de surfactantes ou alguns tipos de efluentes industriais é necessário reduzir a contaminação ainda mais, o que pode ser feito pela utilização dos eletrodos modificados por polímeros.

A utilização do NCTMFE na análise BIA-ASV de traços de $\mathrm{Pb}$ e $\mathrm{Cd}$ foi avaliada ${ }^{43}$. O procedimento para a preparação do NCTMFE foi otimizado. A sensibilidade relativa para os eletrodos com e sem cobertura de Nafion foi 0,9 e 0,8 para $\mathrm{Cd}$ e $\mathrm{Pb}$, respectivamente e os limites de detecção foram $2 \mathrm{nmol} \mathrm{L}^{-1}$ e 4 nmol L $\mathrm{L}^{-1}$. A influência de surfactantes foi examinada e destacou-se a proteção pouco eficiente contra Triton X-100 $-5 \mathrm{mg}$ $\mathrm{L}^{-1}$. Aplicações em águas residuárias foram apresentadas.

A determinação de metais traço utilizando-se BIA-ASV com eletrodo RAM (Random Assemblies of Microdisks) de fibra de carbono coberto com Nafion foi examinada por Fungaro et al. ${ }^{44}$. O volume de amostra injetada praticamente não influenciou a corrente de pico do metal na faixa de 20 a $100 \mu \mathrm{L}$ (Figura 4a). Observou-se o efeito da estabilização do filme de $\mathrm{Hg}$ pelo Nafion. A dependência da corrente de pico com a velocidade de injeção da amostra apresentou-se praticamente constante nas velocidades de 24,5 e $47,6 \mathrm{~mL} \mathrm{~s}^{-1}$ e decresceu em 75,3 $\mathrm{mL} \mathrm{s}^{-1}$ (Figura $4 \mathrm{~b}$ ).

As correntes de pico dos metais eram mais baixas em relação ao eletrodo RAM não coberto, particularmente para o $\mathrm{Pb}$, refletindo os limites de difusão através do filme de Nafion. Porém, uma melhor resolução foi atingida entre os componentes da amostra contendo uma mistura de metais. Os limites de detecção foram $3,6 \mathrm{nmol} \mathrm{L}^{-1}$ e $4,7 \mathrm{nmol} \mathrm{L}^{-1}$ para o $\mathrm{Pb}$ e $\mathrm{Cd}$ respectivamente para injeções simples correspondendo a $1,0 \mathrm{nmol} \mathrm{L}{ }^{-1}$ e $1,3 \mathrm{nmol} \mathrm{L}^{-1}$ para quatro injeções consecutivas de $25 \mu \mathrm{L}$ da amostra. O eletrodo RAM coberto com filme de mercúrio e modificado com Nafion reduziu o limite de detecção e melhorou a sensibilidade das respostas BIAASV na determinação de metais traço em água em comparação com o uso de macroeletrodos.

Em geral, o Nafion apresenta pouca eficiência para a exclusão de surfactantes positivamente carregados e surfactantes não iônicos na determinação de metais em amostras reais, conforme estudos anteriores constataram ${ }^{6,31,32,43}$. Brett et al. ${ }^{45}$ estudaram a modificação do eletrodo de trabalho usando a mistura de Nafion com pequenas porcentagens de polímeros vinílicos e estireno sulfonados solúveis em água. A Figura 5 mostra os resultados obtidos com a adição de Triton X-100 em amostras de $\mathrm{Cd}$ e $\mathrm{Pb}$ nos vários tipos de coberturas de polímeros.

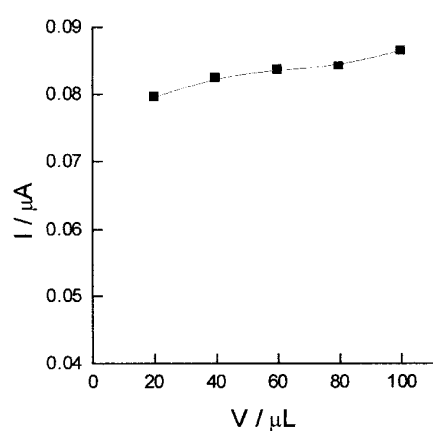

(a)

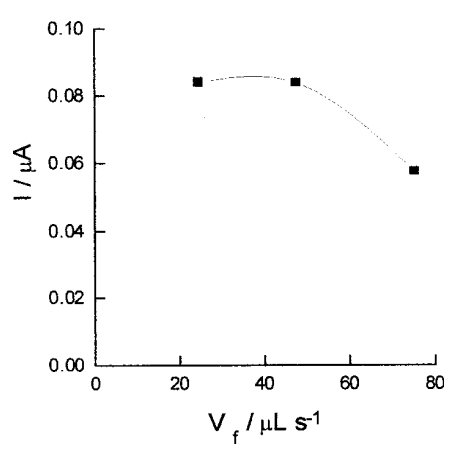

(b)

Figura 4. BIA-ASV do $\mathrm{Pb}^{10^{-7}} \mathrm{~mol} \mathrm{~L}^{-1}$ em $\mathrm{KNO}_{3} 0,10 \mathrm{~mol} \mathrm{~L}^{-1} / \mathrm{HNO}_{3} 5$ mmol $L^{-1} ; E_{\text {dep. }}=-1,0$ V e $t_{\text {dep. }}=30 \mathrm{~s}$. (a) Efeito do volume de injeção na corrente de pico (b) Efeito da velocidade de fluxo na corrente de pico.

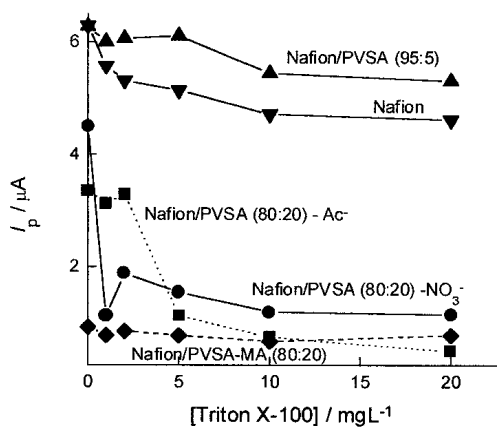

(a)

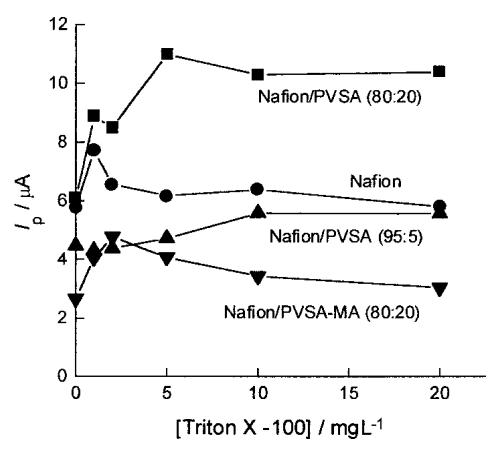

(b)

Figura 5. Dependência da corrente de pico em função da concentração de Triton X-100 para (a) Cd $10^{-7} \mathrm{~mol} \mathrm{~L}^{-1}$ e (b) $\mathrm{Pb} 10^{-7} \mathrm{~mol} \mathrm{~L} \mathrm{~L}^{-1}$, em meio de nitrato e acetato. Eletrodos cobertos com Nafion e: poli (ácido sulfônico vinílico) - PVSA; poli (ácido sulfônico vinílico-coanidrido maleíco) - PVSA-MA. 
Tabela 2. Resultados das curvas de calibração das determinações do Pb e Cd usando-se BIA-ASV e sensor modificado com diferentes dispersões de Eastman AQ.

\begin{tabular}{|c|c|c|c|c|c|}
\hline Dispersão do AQ & & $\begin{array}{l}\text { Sensibilidade } \\
\left(\mu \mathrm{A} / \mathrm{nmol} \mathrm{L}^{-1}\right)\end{array}$ & $\begin{array}{c}\text { Interseção } \\
(\mu \mathrm{A}) \\
(\%)\end{array}$ & $\begin{array}{l}\text { Coeficiente de } \\
\text { Correlação } \\
\left.(\mathrm{nmol} \mathrm{L})^{-1}\right)\end{array}$ & $\begin{array}{r}\text { Limite de } \\
\text { detecção }\end{array}$ \\
\hline \multirow[t]{2}{*}{$\begin{array}{l}0,93 \%{ }^{*} \text { em } 1: 2 \\
\text { água/acetona }\end{array}$} & $\mathrm{Cd}$ & 0,021 & 0,067 & 99,4 & 4,8 \\
\hline & $\mathrm{Pb}$ & 0,011 & 0,064 & 99,4 & 5,5 \\
\hline \multirow[t]{2}{*}{$\begin{array}{l}0,25 \%{ }^{*} \text { em } 1: 2 \\
\text { água/acetona }\end{array}$} & $\mathrm{Cd}$ & 0,0034 & 0,012 & 99,5 & 3,4 \\
\hline & $\mathrm{Pb}$ & 0,0014 & 0,0024 & 99,6 & 5,9 \\
\hline \multirow{2}{*}{$\begin{array}{l}0,25 \% \%^{*} \text { em } 5: 1 \\
\text { água/acetona }\end{array}$} & $\mathrm{Cd}$ & 0,012 & 0,0065 & 99,7 & 3,7 \\
\hline & $\mathrm{Pb}$ & 0,024 & 0,089 & 99,7 & 3,8 \\
\hline
\end{tabular}

(*) $\mathrm{m} / \mathrm{v}$

O eletrodo coberto com o filme da mistura de $95 \%$ Nafion e 5\% de poli (ácido sulfônico vinílico) mostrou o melhor resultado na determinação BIA-ASV de metais na presença de Triton X-100 (Fig. 5), dodecil sulfato de sódio e proteína padrão. Os procedimentos foram aplicados na análise de amostras de água de rio e efluentes industriais.

As características do sensor eletroquímico de carbono vítreo modificado com o polímero Eastman AQ utilizado em BIA-ASV na análise de metais foram investigadas ${ }^{46}$. As dispersões do polímero em diferentes concentrações foram preparadas em diversas misturas água/acetona. O efeito da composição do filme foi mostrada para a determinação de $\mathrm{Pb}$ e $\mathrm{Cd}$ e os limites de detecção estavam ao redor de $5 \mathrm{nmol} \mathrm{L}^{-1}$ em todos os eletrodos testados (Tabela 2). A discriminação contra surfactantes foi excelente e o Eastman AQ mostrou-se uma alternativa viável ao uso do Nafion, principalmente para a aplicação em amostras ambientais contendo uma grande quantidade de detergentes não-iônico.

Existem poucos estudos sobre a utilização de métodos voltamétricos com eletrodos modificados por polímeros perfluorados e sulfonados para a determinação de espécies orgânicas em amostras ambientais. Geralmente, os métodos foram desenvolvidos para estudos das características eletroquímicas dos compostos e não se mostraram práticos para a aplicação com amostras reais. Só mais recentemente começam a surgir pesquisas enfocando o campo de análises ambientais.

Um dos poucos exemplos de estudo é do método de voltametria de redissolução catódica com NCTMFE desenvolvido para a determinação de nitrosaminas aromáticas em águas ${ }^{47}$. A etapa de pré-concentração envolve a redução do analito por um mecanismo eletroquímico-químico-eletroquímico. Ocorre uma condensação e o produto da reação é reduzido em potencial mais baixo que o da nitrosamina. O limite de detecção foi de $3 \mathrm{nmol} \mathrm{L}{ }^{-1}$ com 10 minutos de pré-concentração do produto de condensação seguida por 5 minutos de préoxidação à $800 \mathrm{mV}$.

Uma outra linha de pesquisa utiliza a mistura dos polímeros perfluorados com ligantes capazes de interagir especificamente com espécies em solução proporcionando pré-concentração por complexação.

Um eletrodo de grafite espectroscópico coberto com Nafion incorporado com 1-(2-piridilazo)-2-naftol (PAN) foi explorado na determinação de $\mathrm{Pb}$ (II). $\mathrm{O}$ eletrodo modificado mostrou melhora da sensibilidade e seletividade para o $\mathrm{Pb}$ (II) em relação ao $\mathrm{Cu}(\mathrm{II})$ quando comparado com o eletrodo modificado apenas com o Nafion, mas a proteção contra os surfactantes foi a mesma para o dois eletrodos ${ }^{48}$.

A determinação de traços de cobre por voltametria de pulso diferencial foi realizada usando eletrodo de carbono vítreo coberto com Nafion/imidazola. O método foi aplicado na determinação de cobre em águas de rio e residuárias ${ }^{49}$.
Um procedimento baseado na determinação por ASV de cobre lábil após a sua acumulação química em um eletrodo de grafite modificado com Nafion/4-acilpirazolina foi descrito ${ }^{50}$. Os resultados sobre a capacidade de complexação e a especiação do cobre em águas de rio foram apresentados.

É importante ressaltar que nestes casos, as propriedades de troca iônica do polímero vão desempenhar um papel auxiliar e a eficiência da pré-concentração não será determinada apenas pelas interações de troca iônica, mas sim por uma mistura de interações.

A utilização deste tipo de eletrodo é adequada quando se deseja o aumento da sensibilidade e da seletividade do sensor para a determinação de uma só espécie contida em uma mistura. Estudos sobre a especiação de metais em águas também são bastante favorecidos.

\section{CONCLUSÕES}

O grande número de estudos realizados abordando a voltametria de troca iônica atestam o seu potencial para análise de espécies em amostras complexas, tais como amostras de águas muito poluídas e efluentes industriais.

As aplicações aqui descritas utilizam as propriedades dos ionômeros perfluorados e sulfonados trocadores de íons de repelir espécies indesejáveis, enquanto permitem a passagem do analito para a superfície do eletrodo. Estas características fazem com que os eletrodos modificados com estes filmes poliméricos sejam utilizados principalmente na determinação dos principais metais traço biodisponíveis em águas e efluentes industriais. A proteção da superfície do eletrodo contra as substâncias orgânicas mais comuns encontradas em águas (matéria húmica e surfactantes) também é um ponto importante para a utilização destes eletrodos como sensores, porque a amostra ambiental pode ser analisada com pouco ou nenhum pré-tratamento.

Em termos de perspectivas futuras, destaca-se a grande potencialidade do uso dos eletrodos modificados com estes ionômeros na determinação de espécies orgânicas de interesse ambiental, principalmente pesticidas, como já indicam os mais recentes trabalhos.

\section{REFERÊNCIAS}

1. Oyama, N.; Anson, F. C.; J. Electrochem. Soc. 1980, $127,247$.

2. Oyama, N.; Shimomura, T.; Shigehara, K.; Anson, F. C.; J. Electroanal. Chem. 1980, 112, 271.

3. Rubbinstein, I.; Bard, A. J.; J. Am. Chem. Soc. 1980 , 102, 6641 .

4. Espenscheid, M. W.; Ghatak-Roy, A. R.; Moore III, R. B.; Penner, R. M.; Szentirmay, M. N.; Martin, C. R.; J. Chem. Soc. Faraday Trans. I 1986, 82, 1051. 
5. Wang, J. In Electroanalytical Chemistry; Bard, A. J., Ed.; Marcell Dekker; New York, 1992; v. 16, p1.

6. Hoyer, B.; Florence, T. M.; Batley, G. E.; Anal. Chem. 1987, 59, 1608.

7. Hoyer, B.; Florence, T. M.; Anal. Chem. 1987, 59, 2839.

8. Wang, J.; Greene, B.; Morgan, C.; Anal. Chim. Acta 1984, $158,15$.

9. Kalcher, K.; Electroanalysis 1990, 2, 419.

10. Hillman, A. R. In Electrochemical Science and Technology of Polymer; Lindorf, R. G., Ed.; Elsevier Applied Science; London, 1987; v. 1, cap. 5 e 6.

11. Madja, M. In Molecular Design of Electrode Surfaces; Murray, R.; Ed.; Wiley; New York, 1992; p 159.

12. Oyama, N.; Ohsaka, T. In Molecular Design of Electrode Surfaces; Murray, R., Ed., Wiley; New York, 1992; p 333.

13. Inzelt, G. In Electroanalytical Chemistry; Bard, A. J., Ed.; Marcell Dekker; New York, 1994; v. 18, p 90.

14. Forster, R. J.; Vos, J. G. In Analytical Voltammetry; Smyth, M. R.; Vos, J. G, Ed.; Elsevier; Amsterdam, 1992; cap. 7.

15. Arrigan, D. W. M.; Analyst 1994, 119, 1953.

16. Helfferich, F.; Ion Exchange; McGraw -Hill; New York, 1962.

17. Koryta, J.; Dvorak, J.; Principles of Electrochemistry; Wiley; New York, 1962.

18. Ugo, P.; Moretto, L. M.; Electroanalysis 1995, 7, 1105.

19. Weber, J.; Janda, P.; Kavan, L.; Jegorov, A.; J. Electroanal. Chem. 1988, 200, 379.

20. Moore III, R. B.; Martin, C. R.; Macromolecules 1988, $21,1334$.

21. Goebel, G.; Aldebert, P.; Pireni, M.; Macromolecules 1987, 20, 1425.

22. Hoyer, B.; Jensen, N.; Talanta 1994, 41, 449.

23. Striebel, K. A.; Scherer, G. G.; Hass, O.; J. Electroanal. Chem. 1991, 304, 298.

24. Shimazu, K.; Kita, H.; Kuwana, T.; J. Electroanal. Chem. 1989, 258, 49.

25. Abruna, H. D. In Electroresponsive Molecular and Polymeric Systems; Skotheim, T. A., Ed.; Marcel Dekker; New York, 1988; v. 1, p 1.

26. Yeo, R. S.; Yeager, H. L. In Modern Aspects of Electrochemistry; Conway, B. E.; White, R. E.; Bockris, J. O'M., Ed.; Plenum Press; New York, 1985; v. 16, p 437.
27. Martin, C. R.; Rhodes, T. A.; Ferguson, J. A.; Anal. Chem. 1982, 54, 1639.

28. Dunsch, L.; Kavan, L.; Weber, J.; J. Electroanal. Chem. 1990, 280, 313.

29. Morrison, G. M. P.; Florence, T. M.; Anal. Chim. Acta 1988, 209, 97.

30. Morrison, G. M. P.; Florence, T. M.; Electroanalysis 1989, 1,485 .

31. Dalangin, R. R.; Gunasingham, H.; Anal. Chim. Acta 1994, $291,81$.

32. Dam, M. E. R.; Thomsen, K. N.; Pickup, P. G.; Shroder, K. H.; Electroanalysis 1995, 7, 70.

33. Capelo, S.; Mota, A. M.; Gonçalves, M. L. S.; Electroanalysis 1995, 7, 563.

34. Dam, M. E. R.; Shroder, K.; Electroanalalysis 1996, 8, 1040.

35. Matysik, F-M.; Matysik, S.; Brett, A. M. O.; Brett, C. M. A.; Anal. Chem. 1997 69, 1651.

36. Wang, J.; Taha, Z.; Electroanalysis 1990, 2, 383.

37. Ugo, P.; Moretto, L. M.; Mazzocchin, G. A.; Anal. Chim. Acta 1993, 305, 74 .

38. Ugo, P.; Moretto, L. M.; Mazzocchin, G. A.; Anal. Chim. Acta 1993, 273, 229.

39. Wang, J.; Taha, Z.; Anal. Chem. 1991, 63, 1053.

40. Wang, J.; Taha, Z.; Anal. Chim. Acta 1991, 252, 215.

41. Brett, C. M. A.; Brett, A. M. O.; Mitoseriu, L. C.; Electroanalysis 1995, 7, 225.

42. Wang, J.; Lu, J.; Chen, L.; Anal. Chim. Acta 1992, 259, 123.

43 Brett, C. M. A.; Brett, A. M. O.; Matysik, F-M.; Matysik, S.; Kumbhat, S.; Talanta 1996 43, 2015.

44. Fungaro, D. A.; Brett, C. M. A.; Anal. Chim. Acta 1999, $385,257$.

45. Brett, C. M. A.; Fungaro, D. A.; Morgado, J .M.; Gil, M. H.; J. Electroanal. Chem. 1999, 468, 26.

46. Brett, C. M. A.; Fungaro, D. A.; Talanta 2000, 50, 1223.

47. Gorski, W.; Cox, J. A.; Anal. Chem. 1992, 64, 2710.

48. Hu, Z. M.; Seliskar, C. J.; Heineman, W. R.; Anal. Chim. Acta 1998, 369, 93.

49. Nakabayashi, Y. ; Imai, H.; Bull. Chem. Soc. Japan 1994, $67,113$.

50. Labuda, J.; Vanickova, M.; Uhlemann, E.; Mickleer, W.; Anal. Chim. Acta 1994, 284, 517. 